\section{Solitary Gluteus Maximus Muscle Metastasis in a Breast Cancer Patient}

Jun Yong Lee ${ }^{1}$, Hae Won Kang ${ }^{2}$, Sung-No Jung ${ }^{3}$ Department of Plastic and Reconstructive Surgery, ${ }^{1}$ Incheon St. Mary's Hospital, College of Medicine, The Catholic University of Korea, Incheon; ${ }^{2}$ Yeouido St. Mary's Hospital, College of Medicine, The Catholic University of Korea, Seoul; ${ }^{3}$ Uijeongbu St. Mary's Hospital, College of Medicine, The Catholic University of Korea, Uijeongbu, Korea

Correspondence: Sung-No Jung

Department of Plastic and Reconstructive Surgery, Uijeongbu St. Mary's Hospital, College of Medicine, The Catholic University of Korea, 271 Chenboro, Uijeongbu 11765, Korea

Tel: +82-31-820-3576, Fax: +82-31-847-0301

E-mail: jsn7190@catholic.ac.kr

No potential conflict of interest relevant to this article was reported.

Received: 30 Apr 2015・Revised: 16 Jun 2015 • Accepted: 2 Jul 2015 pISSN: 2234-6163 • elSSN: 2234-6171

http://dx.doi.org/10.5999/aps.2015.42.5.661 • Arch Plast Surg 2015;42:661-663 Copyright (C) 2015 The Korean Society of Plastic and Reconstructive Surgeons This is an Open Access article distributed under the terms of the Creative Commons Attribution Non-Commercial License (http://creativecommons.org/licenses/by-nc/3.0/) which permits unrestricted non-commercial use, distribution, and reproduction in any which permits unrestricted non-commercial use, distribit
medium, provided the original work is properly cited.

Tumor metastasis into skeletal muscle is uncommon, and is rarely noticeable clinically. Lung cancer and gastrointestinal tumors are the most frequent primary malignancies that metastasize to the skeletal musculature, but some reports have described muscle metastases from breast cancer [1]. The authors present a case of gluteal muscle metastasis in a patient with a known primary breast cancer.

A 53-year-old woman presented with a mass on her right buttock with pain that was aggravated by walking. She had previously undergone modified radical mastectomy due to left breast cancer. A histopathological examination at the time had shown invasive ductal carcinoma without axillary lymph node metastasis. She had received five cycles of adjunctive chemoradiotherapy with cyclophosphamide, methotrexate, and 5-fluorouracil, followed by tamoxifen. Seven years after surgery, a slowly growing mass developed on her right buttock with mild throbbing pain. On physical examination, tenderness was observed, but no palpable mass was found in the right buttock. A computed tomography (CT) examination of the pelvis found a poorly defined, mildly enhanced intramuscular mass measuring $3 \mathrm{~cm}$ in the patient's right gluteal maximus muscle (Fig. 1), and a subsequent needle biopsy of the lesion confirmed a metastatic, poorly differentiated invasive ductal carcinoma. In order to address suspicion of systemic metastasis, the patient underwent $\mathrm{CT}$ scans of the breast, positron emission tomography-CT, and bone scans. No other metastatic sites were found (Fig. 2). A wide excision and local flap coverage were performed to remove the metastatic mass along with tissue from the gluteus muscle (Fig. 3). Histological findings showed metastatic invasive ductal carcinoma (Fig. 4). She received adjunctive chemotherapy with capecitabine and no local recurrence was observed 12 months after surgery.

Skeletal muscle metastasis of a primary malignancy is relatively rare and can be misdiagnosed as a primary soft tissue sarcoma; however, distinguishing between the two diagnostic possibilities is critical, because the treatment and prognosis may differ markedly [1].

Metastases of breast cancer may appear in any organ, and although different relapse sites may be observed depending on the subtype of breast cancer, the most frequent metastasis sites are bone, the liver, the lungs, and the brain [2]. Skeletal muscle metastasis of breast cancer is very rare. The following
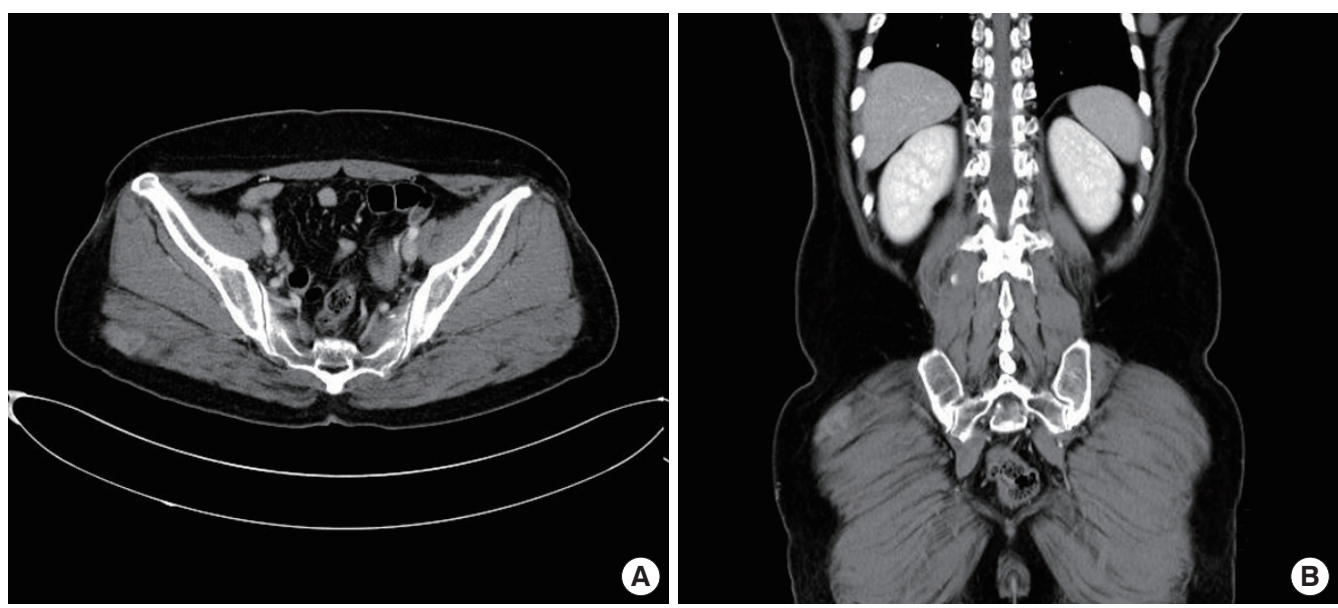

Fig. 1.

(A, B) Preoperative computed tomography views. An enhancing mass is visible in the right gluteus maximus muscle, suggesting an intramuscular metastasis. 


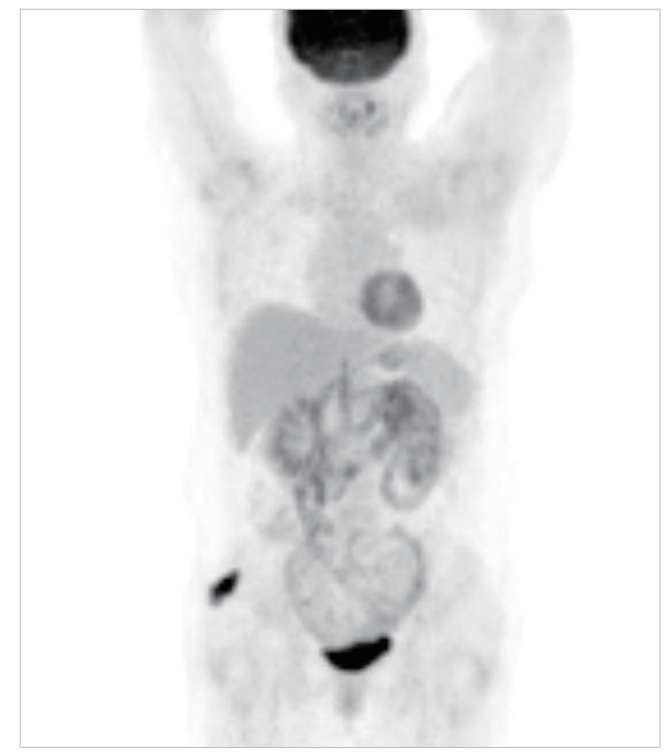

Fig. 2.

Preoperative positron emission tomography-computed tomography (PET-CT) view. PET-CT revealed a significant

hypermetabolic lesion in the right gluteal muscle. No other suspected metastatic sites were found.

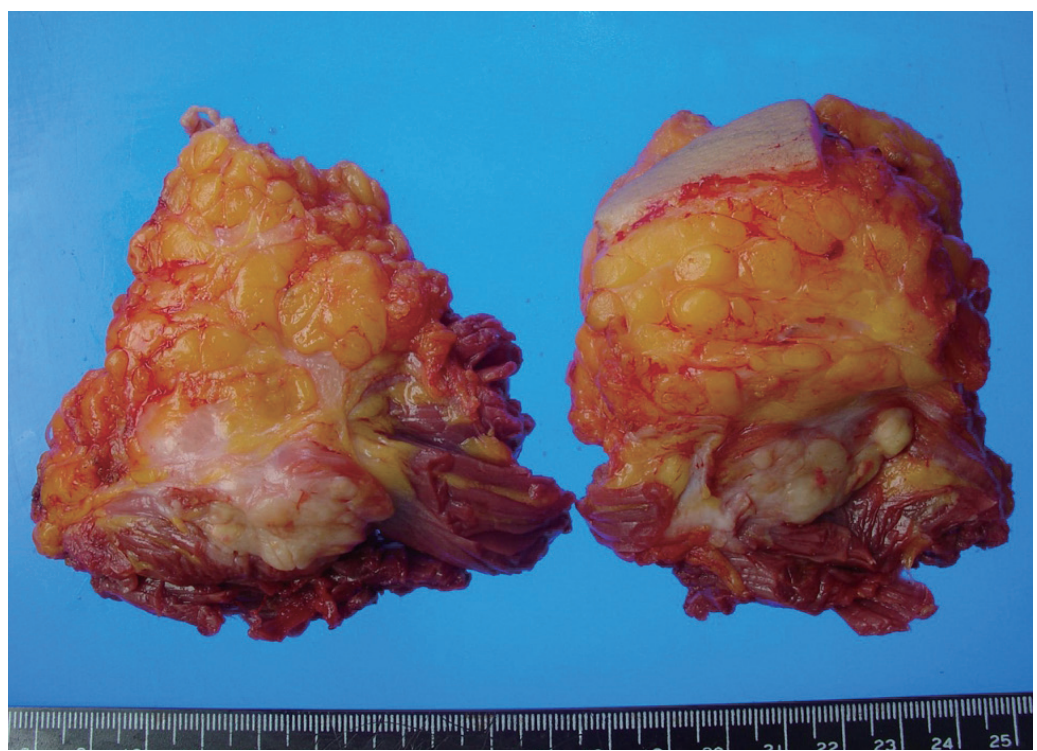

Fig. 3.

Gross photo of the resected intramuscular mass.

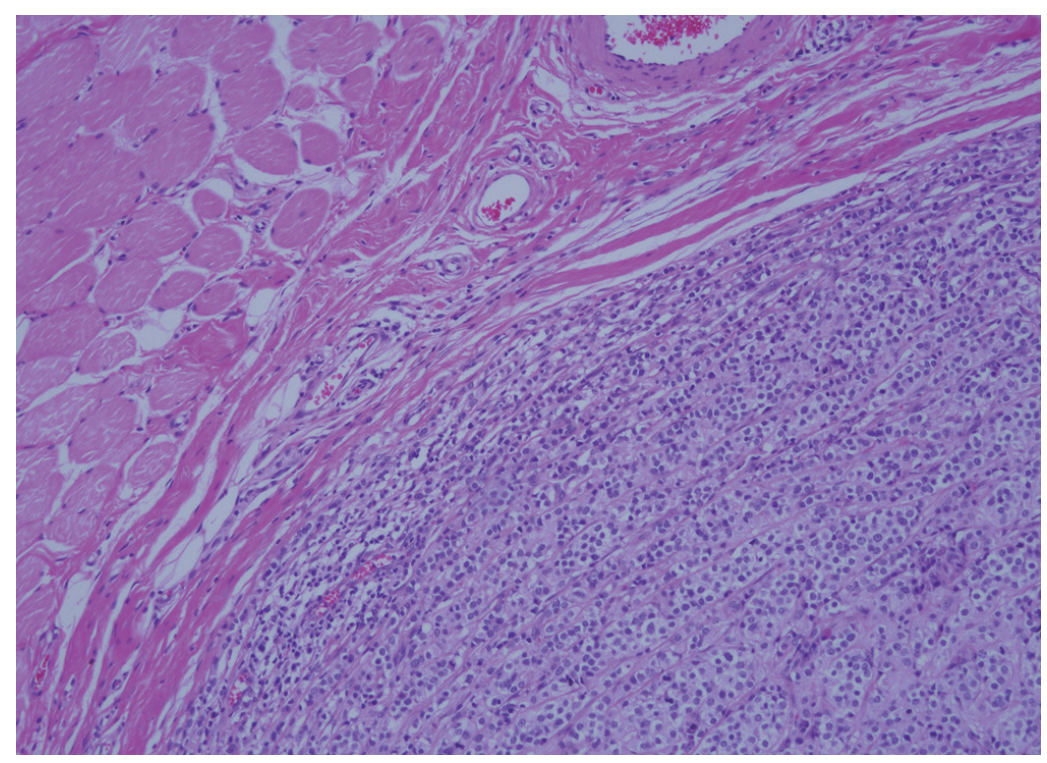

sites have been reported for skeletal muscle metastasis: the abdominal wall muscle, the iliopsoas muscle, the gluteus medius muscle, the triceps muscle, the scalene muscle, the extraocular muscle, the cervical muscle, and the paraspinal muscle [3]. The gluteus maximus muscle has not been reported as a site of skeletal muscle metastasis. It has not been clearly established why tumor metastasis is so rare in skeletal muscle, despite its abundant vascular supply. However, one possible reason is that muscle contractions, in combination with variable but abundant vascular flow, may mechanically prevent tumor cells from stopping and proliferating. It also appears that the settlement and growth of tumors are not favored in muscle tissue due to its $\mathrm{pH}$ value and a chemical environment driving the degradation of lactic acid, an angiogenesis-promoting agent [3].

The most common symptom is pain at the metastatic site, although skeletal muscle metastasis is manifested as the formation of a tumor mass and muscle weakness [3]. If a patient presents with mild pain without clear evidence of a mass upon physical examination, as occurred with the patient in this case, the metastatic tumor may be easily missed. Therefore, careful diagnosis is required.

The prognosis and appropriate treatment of 
skeletal muscle metastasis are uncertain. A previous report recommended that skeletal muscle metastases be treated palliatively if they occur together with systemic metastasis, but an isolated metastatic neoplasm can be resected operatively with or without radiotherapy $[3,4]$.

Although some authors have proposed performing contralateral prophylactic mastectomy, randomized controlled trials to assess the overall survival benefit of this procedure in breast cancer patients have never been carried out, and its efficacy is therefore unclear [5].

This report presented a rare case of a breast cancer metastasis into the gluteal muscle that was completely resected operatively after confirming the diagnosis with a preoperative needle biopsy. In patients with an intramuscular mass, it is necessary to proceed with a suspicion of metastatic cancer, even though metastasis to muscle tissue is rare.

\section{References}

1. Salemis NS. Skeletal muscle metastasis from breast cancer: management and literature review. Breast Dis 2015;35:37-40.

2. Soni A, Ren Z, Hameed O, et al. Breast cancer subtypes predispose the site of distant metastases. Am J Clin Pathol 2015;143:471-8.

3. Ogiya A, Takahashi K, Sato M, et al. Metastatic breast carcinoma of the abdominal wall muscle: a case report. Breast Cancer 2015;22:206-9.

4. Noda S, Kashiwagi S, Kawajiri H, et al. A case of metastatic breast carcinoma of the cervical muscles. Gan To Kagaku Ryoho 2013;40:2405-7.

5. Lostumbo L, Carbine NE, Wallace J. Prophylactic mastectomy for the prevention of breast cancer. Cochrane Database Syst Rev 2010;(11):CD002748.

\section{Endoscopic Excision of Osteochondroma of the Mandibular Angle}

Yeseul Moon, Jai-Kyong Pyon

Department of Plastic Surgery, Samsung Medical Center, Sungkyunkwan University School of Medicine, Seoul, Korea

\section{Correspondence: Jai-Kyong Pyon}

Department of Plastic Surgery, Samsung Medical Center, Sungkyunkwan University School of Medicine, 81 Irwon-ro, Gangnam-gu, Seoul 06351, Korea Tel: +82-2-3410-2235, Fax: +82-2-3410-0036

E-mail: pspriest.pyon@samsung.com

No potential conflict of interest relevant to this article was reported.

Received: 12 Mar 2015• Revised: 15 Apr 2015 • Accepted: 12 May 2015 pISSN: 2234-6163 • elSSN: 2234-6171

http://dx.doi.org/10.5999/aps.2015.42.5.663 • Arch Plast Surg 2015;42:663-665

Copyright (C) 2015 The Korean Society of Plastic and Reconstructive Surgeons

This is an Open Access article distributed under the terms of the Creative Commons

Attribution Non-Commercial License (http://creativecommons.org/licenses/by-nc/3.o/) which permits unrestricted non-commercial use, distribution, and reproduction in any medium, provided the original work is properly cited.

Osteochondroma makes up $35.8 \%$ of all benign bone tumors. It is rare in the craniofacial region [1]. The most common site in the craniofacial region is the mandibular condyle process. In these cases, the mass can result in morphological and functional disturbances. Local resection of the mass is more conservative for treating solitary osteochondroma with a stalk than radial condylectomy and reconstruction $[1,2]$. The surgical approach seems to

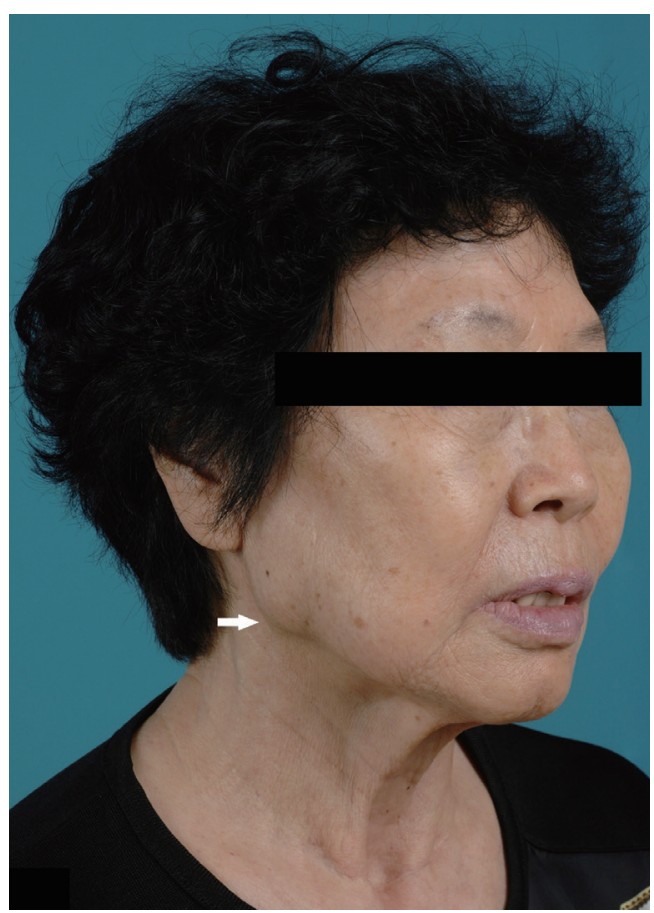

Fig. 1.

Preoperative photograph. An 81-year-old female with a firm mass of the right mandibular angle (white arrow). 